Research Article

\title{
Semiconductor Optical Amplifier Pattern Effect Suppression Using Optical Notch Filtering
}

\author{
Z. V. Rizou ${ }^{1}$, K. E. Zoiros ${ }^{1, *}$ and M. J. Connelly ${ }^{2}$ \\ ${ }^{1}$ Department of Electrical and Computer Engineering, Democritus University of Thrace, Xanthi-67100, Greece. \\ ${ }^{2}$ Department of Electronic and Computer Engineering, University of Limerick, Limerick, Ireland.
}

Received 30 June 2015; Accepted 15 January 2016

\begin{abstract}
We propose to employ four optical notch filtering schemes for mitigating the pattern effect in a semiconductor optical amplifier (SOA). The schemes are capable of feasibly and efficiently realizing significant improvements in the patterndependent performance of the SOA.
\end{abstract}

Keywords: Semiconductor optical amplifier, pattern effect, optical notch filters.

\section{Introduction}

The semiconductor optical amplifier (SOA) has become an indispensable element for the development of lightwave networks owing to the attractive properties of multifunctional capability, low power consumption, small footprint, broad gain bandwidth and integrability [1]. However, its use in the direct signal amplification applications [2] of power boosting, in-line amplification and receiver preamplification is limited by its finite carrier lifetime. Thus when the SOA is biased to operate in the deep saturation regime, its dynamical behavior depends on the binary content of the launched data. This phenomenon, which is known as "pattern effect" [3], causes the output of the SOA to depend on the values of the preceding input data bits instead of solely on the current input bit. The negative by-product of this fact is that the amplified data suffer from amplitude fluctuations, which obstructs the use of conventional SOAs in their classical role.

An efficient method to compensate for the pattern effect exploits the uneven broadening of the spectrum of the amplified pulses to longer wavelengths (red shift) [4]. The idea is to suppress these spectrally broadened components in accordance to the extent of their red shift. For this purpose it is necessary to resort to optical filtering [5]. Thus in this paper we address this issue by means of a set of optical notch filtering schemes, which exhibit several common features, such as passive nature, simple structure, affordable cost, compatibility with fiber medium, periodic and tunable transfer function. By describing the principle of operation of these modules, we show how their transfer function has a comb-like form and we specify how it must be controlled in

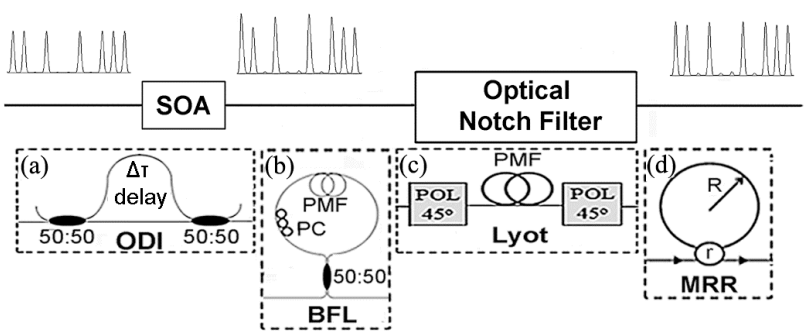

Fig. 1. SOA pattern effect suppresion with optical notch filtering schemes.

order to combat the deleterious consequences of the SOA pattern effect. We present experimental and theoretical results, which demonstrate the considerable improvements achieved in the quality characteristics of the amplified signal as opposed to the SOA alone. Finally, we concisely discuss practical implementation issues of these schemes and further make a qualitative comparison between them in terms of their inherent advantages and disadvantages.

\section{Filtering mechanism for SOA pattern effect suppresion}

Fig. 1 shows the schematic layout of the general configuration. It consists of an SOA followed by an optical notch filter. A random data stream of high peak power and duty cycle is inserted for amplification into the SOA. Under these driving conditions the SOA is heavily saturated and cannot respond timely to this excitation. Consequently the amplified pulses are not uniform but suffer from peak-topeak amplitude fluctuations, as shown right after the SOA.

\footnotetext{
*E-mail address: kzoiros@ee.duth.gr

ISSN: $1791-2377 @ 2016$ Eastern Macedonia and Thrace Institute of Technology. All rights reserved.
} 

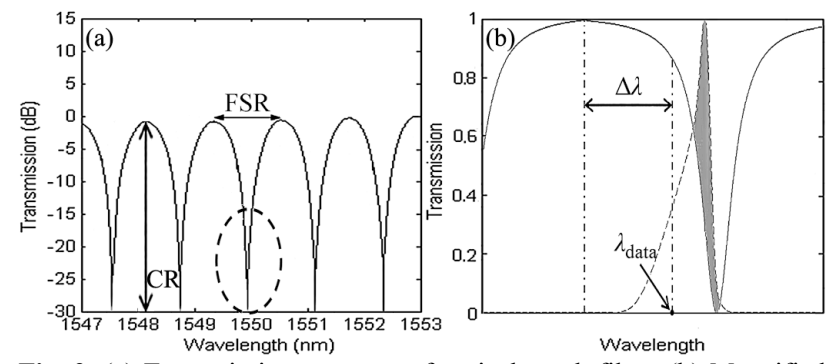

Fig. 2. (a) Transmission response of optical notch filter. (b) Magnified lobe around dashed-circled notch in (a). FSR: Free Spectral Range, defined as the wavelength distance between adjacent maxima. $\Delta \lambda$ : Detuning, defined as the position of the transmission peak with respect to the data carrier wavelength, $\lambda_{\text {data, }}$, so that the amplified red shifted components fall within the grey zone around the notch. CR: Contrast Ratio.

However these pulses are also spectrally broadened to longer wavelengths (red shift) due to self-phase modulation (SPM) that manifests under these operating circumstances [4]. Now since this nonlinear effect is intensity-dependent, the amount of the SPM-induced spectral shift to longer wavelengths is larger for the higher than for the lower amplitude marks. This suggests that the key for resolving the problem of SOA pattern effect is to allow a large part of the broadened spectrum after the SOA to pass for the less intense marks, while block it for the more intense marks. Then the uneven red shift can be compensated and converted into more equalized pulse peak amplitudes. The achievement of this goal requires using some type of optical filtering, and in this context optical notch filters are particularly efficient.

In order for a scheme to act as optical notch filter, its intensity transmission must have the wavelength-dependent profile shown in Fig. 2(a). It consists of alternating maxima and minima, with the null (notch) points situated midway between adjacent peaks, whose wavelength distance defines the free spectral range (FSR). This form allows to cancel the spreading of the spectral components of the amplified pulses towards the longer sideband and accordingly mitigate the SOA pattern-dependent degradation. This can be done if these spectral components are forced to lie around the notches and be suppressed in direct analogy to the degree of their red shift. For this purpose we must properly manipulate the spectral characteristics of the transfer function. This involves choosing and controlling the notches, wavelength spacing (FSR) and detuning according to the following requirements. First, the repetitive notches must be sharp and deep enough so that the relative contrast ratio (CR) between the transmission peak and the adjacent minimum is uniformly maximized across the entire tuning range. Second, the FSR must be such that the transmission peaks are close enough and hence the central wavelength of the input data is more likely to be located in their vicinity. This adjustment allows the amplified signal to pass through the notch filter without significant elimination of useful data information [6]. This requirement is imperative when employing spectral elimination techniques for removing signal impairments [7]. Furthermore, the FSR must be selected so that the falling slope of the spectral lobes is steep enough. This is necessary so that the margin available for the red-shifted spectral components to lie within the grey zone in Fig. 2(b), and hence accumulate near the notches, is sufficiently wide. Moreover, it ensures that these components suffer an attenuation that scales with the uneven peak amplitude of the amplified mark they originate from. This is desirable so that the pattern effect suppression mechanism can function properly. Third, the transmission peak of the spectral response must be set at a smaller wavelength than the optical data carrier, $\lambda_{\text {data, }}$, so that the transmittance is decreased as the wavelength is increased. This is schematically shown in the magnified lobe of Fig. 2(b), where the transmission peak detuning to the shorter sideband of the signal spectrum is denoted by $\Delta \lambda$.

\section{Optical notch filter types}

The conditions specified in the previous section can be satisfied by different types of optical notch filters, which exhibit the desired comb-like transfer function of Fig. 2(a). However these filters differ in their construction and operation and accordingly in the way that this spectral response is obtained and tailored for suppressing the SOA pattern effect. Thus they fall into the following main categories, namely i) intensity-based, with representative the Optical Delay Interferometer (ODI) [8], ii) polarizationbased, with representatives the Birefringent Fiber Loop (BFL) [9] and Lyot [10], and iii) resonator-based, with representative the Microring Resonator (MRR) [11].

The ODI comprises of two $3 \mathrm{~dB}$ polarization maintaining (PM) fused couplers. The output ports of the first coupler are connected to the corresponding straightforward input ports of the second one. In this manner an interferometric configuration is formed whose upper and lower arms have a length difference of equivalent relative time delay, $\Delta \tau$. When the amplified signal is introduced in the ODI from the first coupler, it is split into two beams of equal intensity, which travel along its two arms. Due to their relative delay these beams reach the other end of the ODI at different times. This creates a phase difference between them, $\Delta \varphi=(2 \pi \mathrm{c} \Delta \tau) / \lambda$, where $\mathrm{c}$ is the speed of light in vacuum and $\lambda$ is the reference wavelength. Thus when these beams recombine at the second coupler, the intensity that is produced at the crossed output port varies periodically with $\Delta \varphi$ and subsequently $\lambda$, according to Fig. 2(a). Then the appropriate choice of $\Delta \tau$ permits to tune the ODI through FSR $=\lambda^{2} /(\mathrm{c} \Delta \tau)$ and bias it at the quadrature point of its transmission response, with negative slope versus the optical carrier wavelength [12].

The BFL consists of a PM $3 \mathrm{~dB}$ coupler, a polarization controller (PC) and a segment of polarization maintaining fiber (PMF). Each one of these building components contributes distinctly to the BFL operation. More specifically, the coupler equally splits the incoming amplified signal into two counter-propagating beams, which travel along identical optical paths inside the loop. The PC is set to generate a rotation of $90^{\circ}$ to light coming from both directions so that the orthogonal polarization states, to which the beams are decomposed, are aligned and travel along different axes of the PMF. The PMF of length L introduces via its birefringence $\mathrm{B}$ a relative delay, $\Delta \tau=(\mathrm{BL}) / \mathrm{c}$. As a result the polarization components acquire a phase difference, which is directly proportional to the birefringence and hence wavelength-dependent. Thus when they recombine at the coupler they interfere either constructively or destructively, which produces the spectral response of Fig. 2(a). Notably the exact position of the maxima can be adjusted without affecting the wavelength spacing, FSR $=\lambda^{2} /(\mathrm{BL})$, by rotating the plates of the intraloop PC, which also helps optimize the CR [13].

The Lyot is also composed of a PMF section, which is sandwiched between two polarizers (POL) aligned at $45^{\circ}$ 

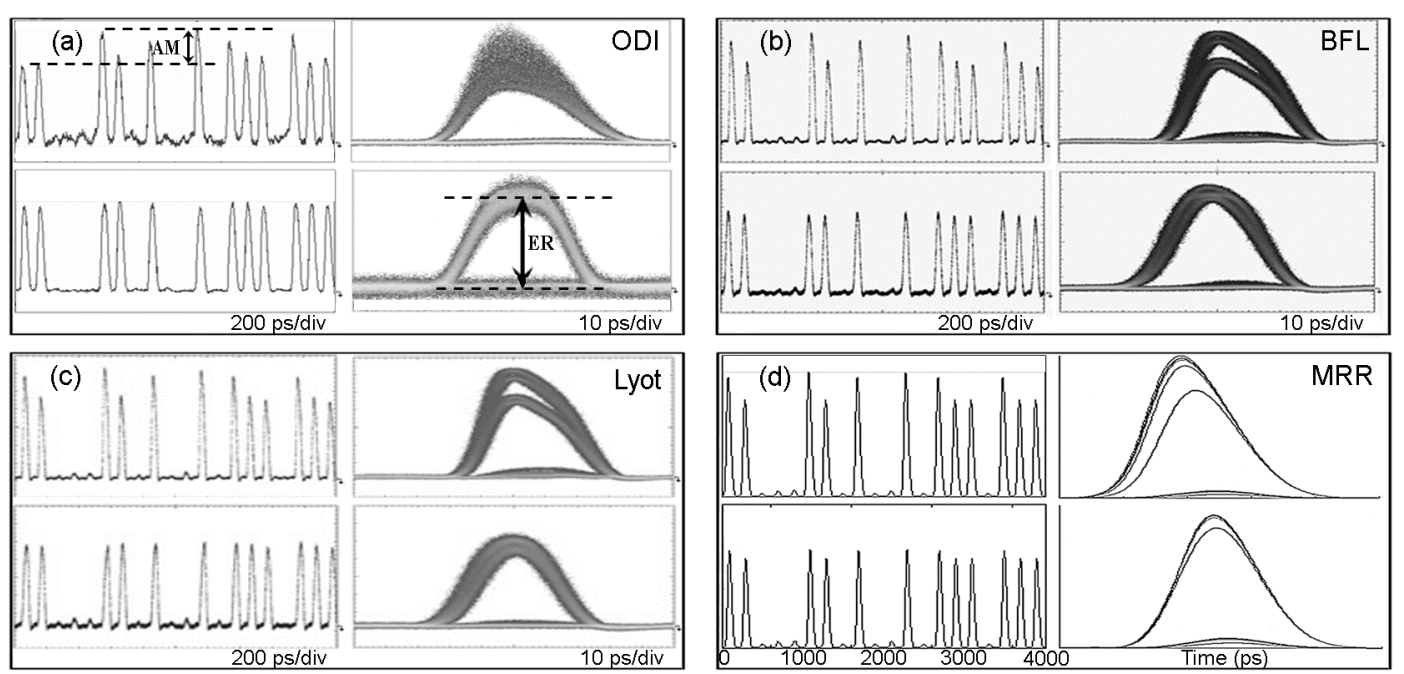

Fig. 3. Resulst for SOA pattern effect suppresion with optical notch filtering implemented using (a) ODI, (b) BFL, (c) Lyot and (d) MRR.

with respect to the birefringent axes of the PMF. Then according to the same principle of operation as for the BFL, the intensity transmission of the Lyot filter matches that of Fig. 2(a). The wavelength shift of a spectral peak up to $50 \%$ of the FSR is controlled by [10] rotating quarter and half wave plates (not shown) right before the first polarizer. The rotation of these plates induces a phase shift, $\Delta \varphi^{\prime}$, which is linked to the detuning through $\Delta \lambda=\left(\Delta \varphi^{\prime} / 2 \pi\right)$ FSR [14].

The MRR is a waveguide shaped into a ring structure that is coupled to a bus waveguide, as shown in Fig. 1(d). When a signal enters into the configuration, part of it is fed back to the input while the rest is transferred at the output. The exact signal magnitude and wavelength that penetrates into the ring depends on the strength of coupling between the straight and circular waveguides and whether the condition of resonance is satisfied. The latter dictates that $2 \pi \mathrm{Rn}=m \lambda_{\text {res }}$, where $\mathrm{R}$ is the ring radius, $\mathrm{n}$ is the refractive index of the guiding material, $\lambda_{\text {res }}$ is the wavelength of the resonating mode and $\mathrm{m}$ is a non-zero integer [15]. The signal components that enter the ring in accordance to these conditions travel around the existing medium and after a time delay of $\Delta \tau=2 \pi \mathrm{Rn} / \mathrm{c}$ interfere with those components that pass directly to the exit. Then provided that the MRR operates in the so-called critical coupling regime [15], where the microring internal losses are equal to the coupling losses, the circulating intensity is maximized and the transmitted intensity becomes minimum. As a result, the spectral response obtains the form depicted in Fig. 2(a), where the free spectral range is FSR $=\lambda^{2} /(2 \pi \mathrm{nR})$ [15]. Moreover, by adjusting the relative position of the notches that occur at resonance through, for example, thermo-optical means, the required negative detuning within half the FSR can be realized for suppressing the SOA pattern effect [11].

\section{Results}

By properly exploiting the characteristics of the optical notch filters described in the previous section, we managed to verify their capability to efficiently suppress the SOA pattern effect. This goal was achieved either through experiment (a-c), or simulation (d). The accomplished performance improvements were monitored with the help of data pulse patterns and eye diagrams, while they were quantified by the amplitude modulation (AM) and extinction ratio (ER). These metrics, which are indicated in Fig. 3(a), should be as low and as high as possible, respectively. In all cases the launched signal was a $10 \mathrm{~Gb} / \mathrm{s}$ return-to-zero $2^{7}-1$ bit-long pseudo random data stream. This signal comprised of pulses that had an initial AM of $0.35 \mathrm{~dB}$ and an ER ranging from $15.6 \mathrm{~dB}$ (a) to $20 \mathrm{~dB}(\mathrm{~b}-\mathrm{d})$. Moreover the pulses had a power and temporal content that brought the SOA into heavy saturation, thereby provoking an inadmissible AM and ER

Fig. 3 shows the results that were obtained, experimentally with the ODI (a), BFL (b) and Lyot (c), and theoretically with the MRR (d). The values of the critical parameters and measurands were $\Delta \tau, \mathrm{FSR}, \mathrm{CR}=$ (a) $1.48 \mathrm{ps}$, $5.4 \mathrm{~nm}, 14 \mathrm{~dB}$, (b) $8.8 \mathrm{ps}, 0.91 \mathrm{~nm}, 20.4 \mathrm{~dB}$, (c) $6.7 \mathrm{ps}, 1.2$ $\mathrm{nm}, 24 \mathrm{~dB}$ and (d) $0.83 \mathrm{ps}, 9.68 \mathrm{~nm}, 24 \mathrm{~dB}$. In this manner the $\mathrm{AM}$ was reduced from $1.6 \mathrm{~dB}(\mathrm{a}), 1.7 \mathrm{~dB}(\mathrm{~b}$ and $\mathrm{c})$ and $1.28 \mathrm{~dB}(\mathrm{~d})$ after the SOA, down to $0.29 \mathrm{~dB}$ (a and b), 0.35 $\mathrm{dB}$ (c) and $0.47 \mathrm{~dB}$ (d), owing to these schemes, respectively. The restoration of the AM to acceptable levels allowed to smooth the peak amplitude excursions and made the profile of the output signal resemble more that of the incoming one. Similarly the ER was increased from $10.5 \mathrm{~dB}$ (a), $10 \mathrm{~dB}$ (b and c) and $10.5 \mathrm{~dB}$ (d), to $13.1 \mathrm{~dB}(\mathrm{a}), 18 \mathrm{~dB}$ (b), $17 \mathrm{~dB}$ (c) and $18.1 \mathrm{~dB}(\mathrm{~d})$. This increase reshaped the eye diagrams, which became close replicas of those at the SOA input.

\section{Qualitative comparison between different optical notch filters}

From the above results it can be realized that the different types of employed optical notch filters show equivalent capability in confronting the SOA pattern effect problem. This means that they provide equal number of technological options to select from for improving the performance of SOAs in direct signal amplification applications. Still there is no absolute criterion for preferring one type to another. Thus making a selection that best suits the particular needs of a SOA-based lightwave link or network requires considering some issues of real operation and implementation of these filters. For this purpose we make first a qualitative comparison between the ODI, BFL and Lyot because their principle of operation is conceptually similar, as it relies on splitting, delaying and recombining the amplified signal. However this process is realized by means of either intensity or polarization discrimination in 
the ODI and BLF-Lyot, respectively. This is a fundamental difference between the ODI and BLF-Lyot, which has the following practical implications. More specifically, the fiber-based and the waveguide-based versions of the ODI are sensitive to environmental perturbations and to polarization deviations, respectively [16]. As a consequence active setting of the bias point, precise phase maintenance and continuous polarization control are necessary in order to achieve and maintain the required condition of interference, at the price of increased complexity. In contrast the BFL with its counter-propagating geometry is inherently more stable, can be made resistant to ambient changes in a more affordable way, and is independent of the input polarization [16]. The Lyot also features similar properties, which as in the BFL are enabled by the existence of common, instead of distinct in the ODI, travelling paths for the split constituents of the incoming signal. Furthermore, building and setting the Lyot is simpler than the BFL owing to the direct-wise structure of the former. But in terms of stability, the conducted experiments [9]-[10] reveal that the Lyot is inferior to the BFL, mainly due to its single branch architecture. Finally, both BFL and Lyot can be conveniently constructed from commercially available bulk components. However this fact has two inevitable drawbacks. First, it results in enlarged physical size, and second it incurs increased insertion losses. On the contrary the ODI is compatible with the planar waveguide technology [17] and so it can be made to have a small footprint. Moreover, it can be monolithically integrated with the SOA in the same module [18], thereby allowing a sufficient margin of net gain for direct signal amplification applications with mitigated SOA pattern effect. On other hand, due the interference of only two copies of the input signal these three schemes have a low finesse. This fact limits their capability to simultaneously handle multiple data channels. This constraint as well as the aforementioned ones can be overcome by the MRR. Compared to the other schemes utilized for the same purpose, the MRR-based notch filter features structural simplicity, ultra compact and small size, compatibility with microelectronic fabrication processes, better wavelength selectivity, enhanced fine tuning capability and contrast ratio, improved operating stability and availability of different material systems [15]. Owing to these attractive characteristics it constitutes an excellent alternative for efficiently combating the SOA pattern effect.

\section{Conclusion}

In conclusion, we have presented how four different schemes can be configured as optical notch filters to suppress the SOA pattern effect. Each scheme has unique features and is capable of efficiently dealing with the specific impairment. Therefore these schemes can establish a technological platform destined to assist the employment of SOAs as direct amplification elements with high performance.

This paper was presented at Pan-Hellenic Conference on Electronics and Telecommunications - PACET, that took place May 8-9 2015, at Ioannina Greece.

\section{References}

1. J. Mørk, M. L. Nielsen, and T. W. Berg, "The dynamics of semiconductor optical amplifiers," Opt. Photon. News, Vol. 14, No. 7, pp. 42-48, Jul. 2003.

2. M. J. Connelly, Semiconductor Optical Amplifiers. Dordrecht, The Netherlands: Kluwer, 2002, pp. 108-120.

3. R. J. Manning, A. D. Ellis, A. J. Poustie, and K. J. Blow, "Semiconductor laser amplifiers for ultrafast all-optical signal processing," J. Opt. Soc. Am. B, Vol. 14, No. 11, pp. 3204-3216, Nov. 1997.

4. G. P. Agrawal and N. A. Olsson, "Self-phase modulation and spectral broadening of optical pulses in semiconductor laser amplifiers," IEEE J. Quantum Electron., Vol. 25, No. 11, pp. 2297-2306, Nov. 1989.

5. K. Inoue, "Optical filtering technique to suppress waveform distortion induced in a gain-saturated semiconductor optical amplifier," Electron.Lett., Vol. 33, No. 10, pp. 885-886, May 1997.

6. T.Watanabe, H. Yasaka, N. Sakaida, and M. Koga, "Waveform shaping of chirp-controlled signal by semiconductor optical amplifier using Mach-Zehnder frequency discriminator," IEEE Photon. Technol. Lett., Vol. 10, No. 10, pp. 1422-1424, Oct. 1998.

7. J. Dong, X. Zhang, F. Wang, W. Hong, and D. Huang, "Experimental study of SOA-based NRZ-to-PRZ conversion and distortion elimination of amplified NRZ signal using spectral filtering," Opt. Commun., Vol. 281, No. 22, pp. 5618-5624, Nov. 2008.

8. K. E. Zoiros, C. L. Janer, and M. J. Connelly, "Semiconductor optical amplifier pattern effect suppression for return-to-zero data using an optical delay interferometer," Opt. Eng., Vol. 49, No. 8, 085005, Aug. 2010.

9. K. E. Zoiros, C. O’Riordan, and M. J. Connelly, "Semiconductor optical amplifier pattern effect suppression using a birefringent fiber loop," IEEE Photon. Technol. Lett., Vol. 22, No.4, pp. 221223, Feb. 2010.
10. K. E. Zoiros, C. O'Riordan, and M. J. Connelly, "Semiconductor optical amplifier pattern effect suppression using Lyot filter," Electron. Lett., Vol. 45, No. 23, pp. 1187-1188, Nov. 2009.

11. Z. V. Rizou, K. E. Zoiros, and A. Hatziefremidis, "Semiconductor optical amplifier pattern effect suppression with passive single microring resonator-based notch filter," Opt. Commun., Vol. 329, pp. 206-213, Oct. 2014.

12. D. Derickson, Fiber Optic Test and Measurement. New Jersey, USA: Prentice Hall Professional Technical Reference, 1997, ch. 5 .

13. D. Liu, N. Q. Ngo, H. Liu, and D. Liu, "Stable multiwavelength fiber ring laser with equalized power spectrum based on a semiconductor optical amplifier," Opt. Commun., Vol. 282, No. 8, pp. 1598-1601, Apr. 2009.

14. M. Wang, S. Fu, P. Shum, N. Q. Ngo, J. Wu, and J. Lin, "A tunable Lyot birefringent filter with variable channel spacing and wavelength using nonlinear polarization rotation in an SOA,", IEEE Photon. Technol. Lett., Vol. 20, No. 18, pp. 15271529, Sep. 2008.

15. D.G. Rabus, Integrated Ring Resonators: The Compendium. Berlin, Germany: Springer-Verlag, 2007.

16. C. W. Chow, C. S. Wong, and H. K. Tsang, "Reduction of amplitude transients and BER of direct modulation laser using birefringent fiber loop," IEEE Photon. Technol. Lett., Vol. 17, No. 3, pp. 693-695, Mar. 2005.

17. H. Dong, G. Zhu, Q. Wang, H. Sun, N. K. Dutta, J. Jaques, and A. B. Piccirilli, "Multiwavelength fiber ring laser source based on a delayed interferometer", IEEE Photon. Technol. Lett., Vol. 17, No. 2, pp. 303-305, Feb. 2005.

18. A. Bhardwaj, N. Sauer, L. Buhl, W. Yang, L. Zhang, and D. T. Neilson, "An InP-based optical equalizer monolithically integrated with a semiconductor optical amplifier", IEEE Photon. Technol. Lett., Vol. 19, No. 19, pp. 1514-1516, Oct. 2007. 